MATEC Web of Conferences 21, 09002 (2015)

DOI: $10.1051 /$ matecconf $/ 20152109002$

(C) Owned by the authors, published by EDP Sciences, 2015

\title{
Plasma-nitriding assisted micro-texturing into stainless steel molds
}

\author{
Tatsuhiko Aizawa ${ }^{1, a}$, Hiroaki Suga ${ }^{1}$, and Tetsuya Yamaguchi ${ }^{2}$ \\ ${ }^{1}$ Department of Engineering and Design, Shibaura Institute of Technology, 108-8548 Tokyo, Japan \\ ${ }^{2}$ R \& D Center, Sanko-Light Industry, Co. Ltd. 211-0053 Kawasaki, Japan
}

\begin{abstract}
Micro-texturing has grown up to be one of the most promising procedures. This related application required for large-area, fine micro-texturing onto the stainless steel mold materials. A new method other than laser-machining, micro-milling or microEDM was awaited for further advancement of this micro-texturing. In the present paper, a plasma nitriding assisted micro-texturing was developed to make various kinds of micropatterns onto the martensitic stainless steels. First, original patterns were printed onto the surface of substrate by using the ink-jet printer. Then, the masked substrate was subjected to high density plasma nitriding; the un-masked surfaces were nitrided to have higher hardness. This nitrided substrate was further treated by sand-blasting to selectively dig the soft, masked surfaces. Finally, the micro-patterned martensitic stainless steel substrate was fabricated as a mold to duplicate these micro-patterns onto the work materials. The spatial resolution and depth profile controllability of this plasma nitriding assisted micro-texturing was investigated for variety of initial micro-patterns. The original size and dimension of initial micro-patterns were precisely compared with the three dimensional geometry of micro-textures after blasting treatment. The plastic cover case for smart cellular phones was employed to demonstrate how useful this processing is in practice.
\end{abstract}

\section{Introduction}

Micro- and nano-textures on the metal and polymer surfaces of parts and components have functions to reduce the friction and wear and to improve the joining strength [1,2]. As needed, the surface texturing is also employed to have the surface profile leather-touched or textile-patterned. In order to fabricate these micro- and nano-textured surface of products, the mold-die units for injection molding must have their original micro- and nano-textures on their surface. When using the micro-milling or micro-EDM, huge amount of digital data has to be built in as CAM data before actual time-consuming micro-milling or micro-EDM [3, 4]. A non-traditional micro-texturing method is demanded in market to fabricate the injection molds in much shorter processing time. The authors [5-9] have proposed an alternative micro-texturing with aid of the high density plasma nitriding.

\footnotetext{
${ }^{a}$ Corresponding author: taizawa@sic.shibaura-it.ac.jp
}

This is an Open Access article distributed under the terms of the Creative Commons Attribution License 4.0, which permits unrestricted use, distribution, and reproduction in any medium, provided the original work is properly cited. 

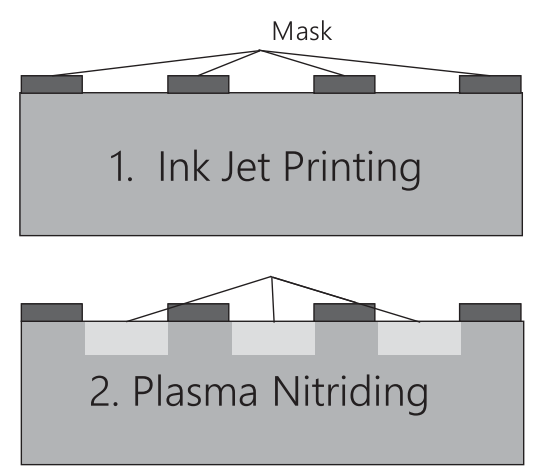
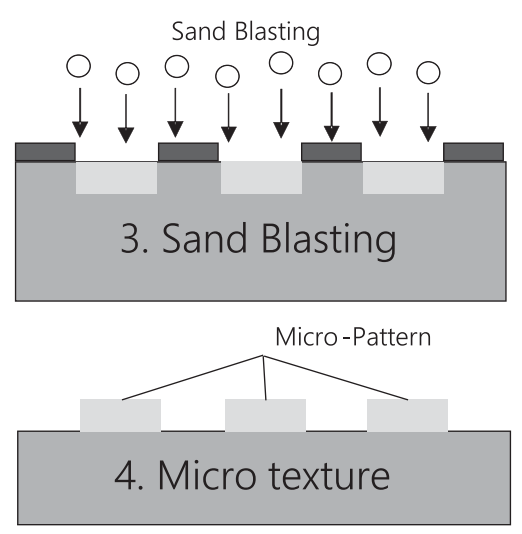

Figure 1. Typical procedure of the plasma nitriding assisted micro-texturing process.

In the present paper, this micro-texturing method is described for each step to fabricate the microtextured stainless steel mold for injection. The ink-jet printing method is first employed to print the two-dimensional micro-patterns directly on the surface of substrate materials. In the following plasma nitriding, this micro-patterns are utilized as a mask for nitriding. The sand-blasting is further used to mechanically remove the masks and to machine the micro-textures into substrate. Finally, the injection molding is utilized to duplicate the micro-textures cut into the mold onto the plastic cover-case surface.

\section{Micro-texturing}

The micro-texturing process is composed of three steps as illustrated in Fig. 1. In the initial micropatterning step, the designed micro-patterns are ink-jet printed directly on the mold surface. This mold is further subjected to plasma nitriding in the second step. In the final step, the nitrided mold is treated by the sand-blasting to fabricate the micro-textured mold.

\subsection{Initial micro-patterning}

The flat-bed ink-jet printer with UV-irradiation instrumentation (Mimaki, Co., Ltd.) was utilized to print the designed micro-patterns directly onto the AISI420 test-pieces and molds. The content of inks must be optimized to have sufficient heat resistance to be alive during the plasma nitriding at $693 \mathrm{~K}$. Through the preliminary tests, the compound of polymer primer and carbon black was employed for the ink-jet printing in this study. Figure 2 depicted the top view of printed micro-dot patterns after plasma nitriding for $14.4 \mathrm{ks}$ (or 4 hours). No defects and failure of dot patterns are observed and detected.

\subsection{High density plasma nitriding}

The present high density nitriding system has no mechanical matching box with slow response time of $1 \mathrm{~s}$ to $10 \mathrm{~s}$ to adjust the applied power. Since both the input and output powers are automatically matched by frequency adjustment around $2 \mathrm{MHz}$, the matching response time is only limited to $1 \mathrm{~ms}$ at most. This prompt power control provides to make full use of mesoscopic plasma pressure range over $50 \mathrm{~Pa}$. Different from the conventional processes, the vacuum chamber is electrically neutral so that RFpower and DC-bias should be controlled independently from each other. A dipole electrode is utilized to generate RF-plasma; DC bias is directly applied to the specimens. Heating unit is located under this DCbiased cathode plate. In the following nitriding experiments, the specimens are located on the cathode 


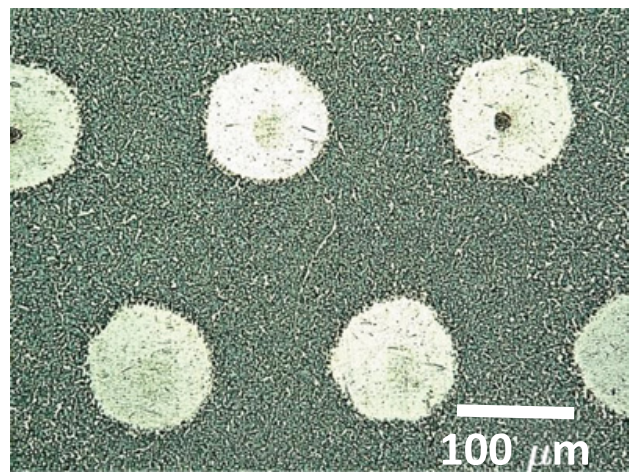

Figure 2. Top view of micro-patterns on the AISI 420 test-piece after plasma nitriding at $693 \mathrm{~K}$ for $14.4 \mathrm{ks}$.

Table 1. Plasma nitriding conditions for micro-texturing.

\begin{tabular}{ll}
\multicolumn{1}{c}{ Process } & \multicolumn{1}{c}{ Parameters } \\
Pre-sputtering & $\mathrm{DC}(500 \mathrm{~V})$ \\
& $\mathrm{N}_{2}(70 \mathrm{~Pa})$ at 693 \\
& $\mathrm{~K}$ for $1.8 \mathrm{ks}$ \\
\hline \multirow{3}{*}{ Nitriding } & $\mathrm{RF}(250 \mathrm{~V})$, \\
& $\mathrm{DC}(300 \mathrm{~V})$ \\
& $\mathrm{N}_{2}(100 \mathrm{ml} / \mathrm{min})$ \\
& $\mathrm{H}_{2}(20 \mathrm{ml} / \mathrm{min})$ \\
& $70 \mathrm{~Pa}$ at $\mathbf{6 9 3 ~ K}$ \\
& for $\mathbf{1 0 . 8} \mathbf{~ k s}$ \\
\hline
\end{tabular}

table before evacuation down to the base pressure of $0.1 \mathrm{~Pa}$. Then, nitrogen gas is first introduced as a carrier gas for heating. After heating to the specified holding temperature, the nitrogen pre-sputtering is started at the constant pressure. After pre-sputtering, the hydrogen gas is added to nitrogen gas with the specified partial pressure ratio. Both pressure $(\mathrm{P})$ and temperature $(\mathrm{T})$ controls are automatically performed with the tolerance of $\Delta \mathrm{P}<1 \mathrm{~Pa}$ in deviation of partial pressure and $\Delta \mathrm{T}<1 \mathrm{~K}$ in temperature fluctuation.

Table 1 summarizes the experimental conditions for present plasma nitriding. In particular, the partial pressure ratio of nitrogen to hydrogen gases is constant by 5 to 1 as a standard condition by controlling the gas flow rate to be $100 \mathrm{ml} / \mathrm{min}$ for $\mathrm{N}_{2}$ gas and $20 \mathrm{ml} / \mathrm{min}$ for $\mathrm{H}_{2}$ gas, respectively. The holding temperature is $693 \mathrm{~K}$ or $420^{\circ} \mathrm{C}$.

\subsection{Sand-blasting}

The air-blasting system type SFC (Fuji-Seiki, Co. Ltd.) was also employed as a blasting system to remove the masked or un-nitrided regions and to polish the mold surface by using the optimum blasting media. In this process, the silica powders with the average diameter of $30 \mu \mathrm{m}$ were used as a blasting medium. Under this selection of blasting media, the soft regions are selectively machined without any damage to the harder regions.

\section{Experimental}

The initially printed two dimensional micro-dot patterns change themselves to the three dimensional hardness profile into the AISI420 test-pieces and molds. Under this selective nitriding and hardening process, the masked or unnitrided regions remain the same hardness as the AISI420 matrix to be 

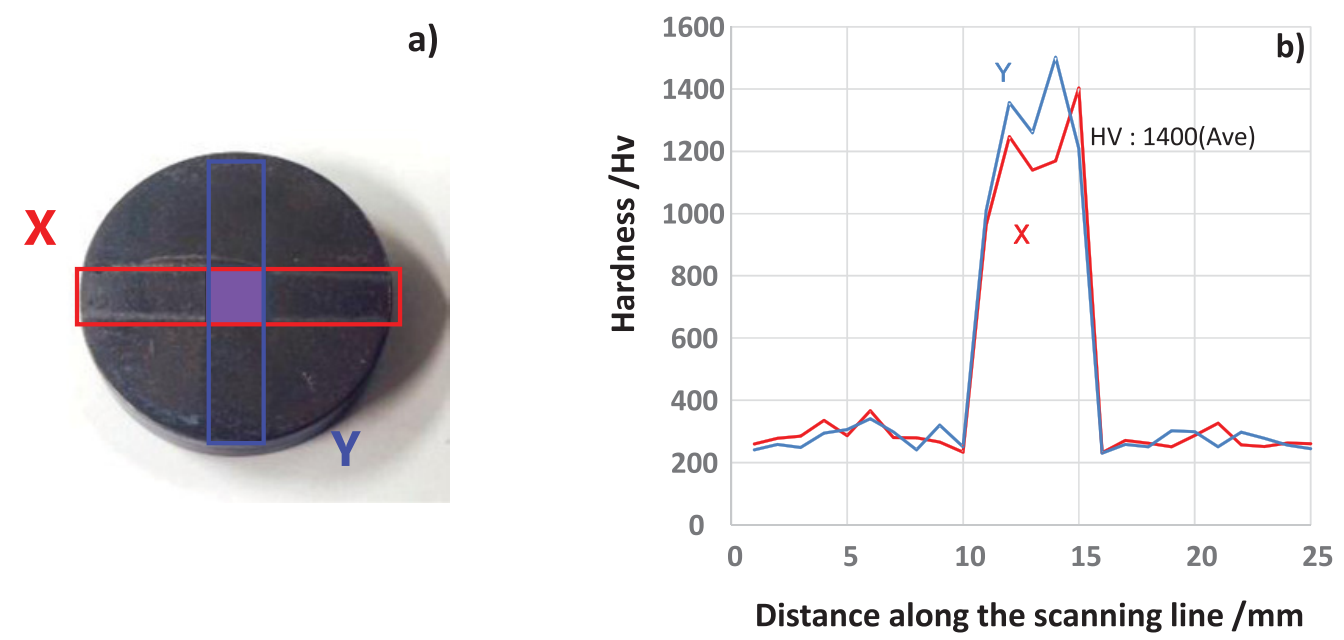

Figure 3. Hardness profile across the nitrided area with small area on the AISI420 specimen. a) Masking configuration on the masked AISI420 specimen, and, b) hardness profiles in the $\mathrm{x}$ - and $\mathrm{y}$-directions in Fig. 3a).

selectively machined into depth by the sand-blasting. This micro-dimpled AISI420 mold is utilized for injection molding to demonstrate that the initial micro-dot pattern is duplicated onto the plastic cover case surface as a micro-disc pattern.

\subsection{Selective nitriding and hardening}

The AISI420 specimen surface except for the square area with around $5 \times 5 \mathrm{~mm}^{2}$ at its center, was masked by printing to make nitriding only on the small area, as shown in Fig. 3a). Figure 3b) depicted the measured hardness profile in the two directions across this printed pattern after plasma nitriding at $693 \mathrm{~K}$ for $7.2 \mathrm{ks}$; i.e. in the $\mathrm{x}$ and $\mathrm{y}$ directions in Fig. 3a). The masked specimen surface has lower hardness of bare stainless steel AISI420, around $250 \mathrm{Hv}$; while the unmasked small area has higher hardness than $1400 \mathrm{Hv}$. Since no significant difference is detected between the hardness profiles measured along the $\mathrm{x}$ and $\mathrm{y}$-directions, this un-masked surface is homogeneously nitrided to have high hardness. Since no $\mathrm{CrN}$ and $\mathrm{Fe}_{4} \mathrm{~N}$ were synthesized by this plasma nitriding, this hardening is attributed to solid solution hardening by nitrogen solute. In Fig. 3, the hardness profile changes itself with steep gradient across the border between the masked and unmasked areas. This implies that little or no nitrogen solute is present in the masked AISI420 specimen and that significantly high content of nitrogen solute exists in the unmasked one.

In order to investigate this difference of nitrogen solute concentration between two regions, the above specimen in Fig. 3a) was wire-cut into halves by EDM (electric discharging machining) to prepare for samples. FE-SEM and EDX were utilized to make observation and nitrogen mapping on the crosssection of specimen. Figure 4 depicts the SEM image of microstructure and the nitrogen solute mapping on this cross-section.

The nitriding front end advances into the depth of specimen in the unmasked region; the nitrided layer thickness is uniform by $40 \mu \mathrm{m}$. On the other hand, this nitrided layer thickness becomes smaller near the vicinity of border between the masked and unmasked regions. Just on the border, this thickness finally approaches to zero. Corresponding to this change of the nitriding front end or the nitrided layer thickness, the nitrogen solute map changes itself when approaching to the border. The nitrogen solute content is high in the unmasked region. The average of measured nitrogen solute content in the middle 

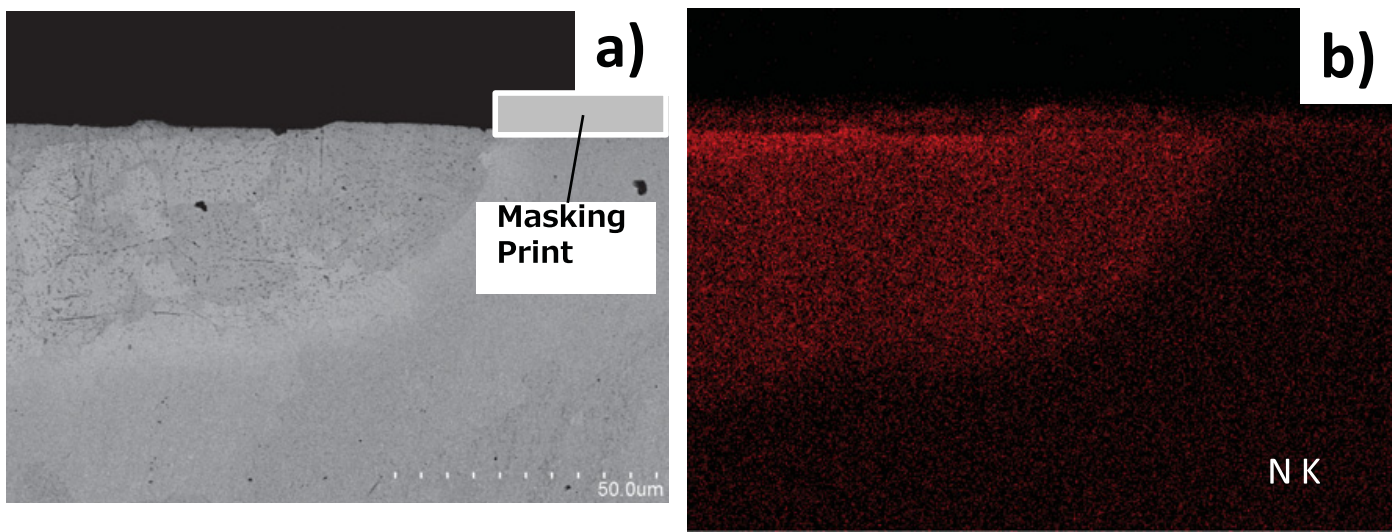

Figure 4. SEM image and nitrogen solute mapping on the cross-section of nitrided AISI420 specimen. a) Microstructure of nitrided layer in the AISI420 substrate, and, b) nitrogen solute map in the AISI420 substrate.
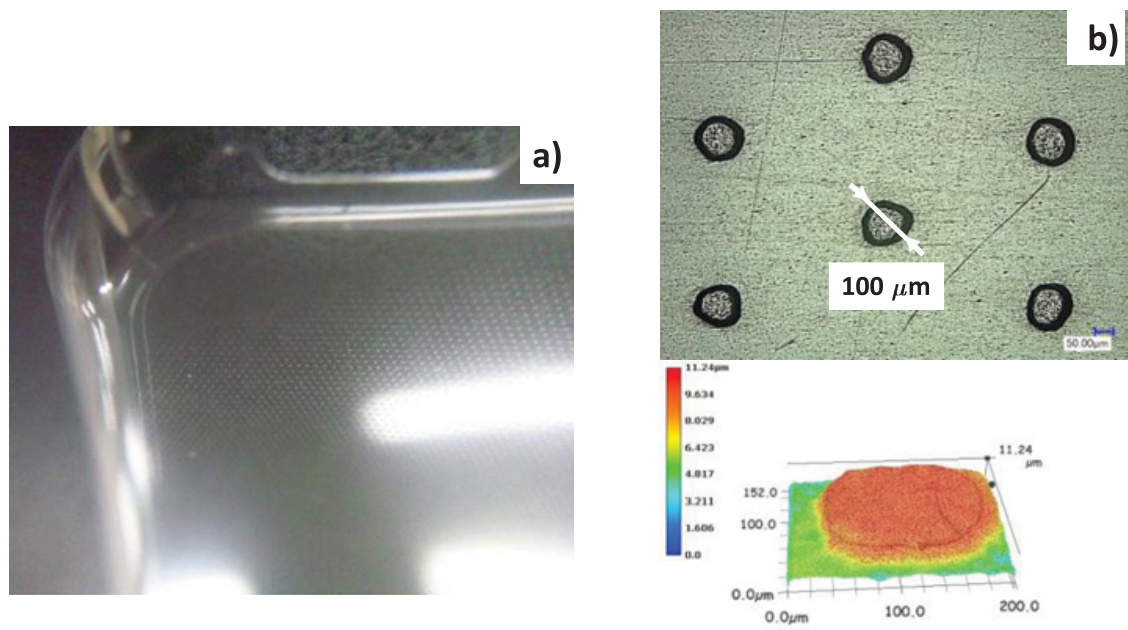

Figure 5. A plastic cover case with micro-disc patterns duplicated onto its surface via the injection molding. a) Outlook of the convex micro-disc patterns on the plastic cover-case, and, b) micro-disk patterns on the cover case.

of nitrided layer is 4 mass $\%$ or 15 at $\%$. This high nitrogen solute map is reduced near the vicinity of the border and disappears just on the border.

The above change of microstructure and nitrogen solute map implies that no nitrogen solute is present and responsible for hardening by solid solution strengthening in the masked region. The steep change of hardness across the border is just corresponding to the change of nitrided layer thickness and nitrogen solute content. This fact reveals that the AISI420 mold substrate just below the printed masks could be removed by the sand-blasting to form micro-dimple patterns in correspondence to the original circular dot-patterns printed on the mold surface.

\subsection{Fabrication of micro-textured molds}

A micro-dimple textured mold-unit was fabricated by the present micro-texturing process. The microdot pattern was ink-jet printed onto the AISI420 mold-unit surface of $120 \times 65 \mathrm{~mm}^{2}$, plasma nitrided at 


\section{MATEC Web of Conferences}

$693 \mathrm{~K}$ for $10.8 \mathrm{ks}$ and sand-blasted by silica powders for $300 \mathrm{~s}$. The micro-dimple texture was machined into a mirror-polished area of mold-die after fine sand-blasting for $60 \mathrm{~s}$. Through the injection molding, the polycarbonate (PC) melt is expected to flow into each micro-dimples as well as a cavity of plastic caver case body. Figure 5 showed a PC cover case for cellular phone with micro-disc patterns. Through the optical microscopy, these micro-discs with the average diameter of $100 \mu \mathrm{m}$ are regularly aligned on the cover-case surface with the pitch of $400 \mu \mathrm{m}$ between their center points, as shown in Fig. 5b). The surface profile measurement reveals that this micro-disc has a flat top surface with standing side surface against the PC body surface. This is because the original micro-dimple machined onto the mold-unit surface has a flat bottom surface and high-gradient side-surface along the edge of micro-dimple pattern.

\section{Conclusion}

The plasma nitriding assisted micro-texturing is proposed as a new method to cut the tailored micropatters into the AISI420 martensitic stainless steel molds as a concave micro-texture to be duplicated as a convex micro-pattern on the plastic product. This process consists of three steps. The ink-jet printing is the first step to draw the original two dimensional micro-patterns directly on the mold surface. Through optimization of the ink contents, the printed patterns have sufficient heat resistivity even during plasma nitriding at $693 \mathrm{~K}$ or $420^{\circ} \mathrm{C}$. The next step is the high density plasma nitriding where the unmasked regions of mold is selectively nitrided and hardened to transform the original printed micro-patterns to the three dimensional hardness profile. The selectively nitrided regions have higher nitrogen solute content of 4 mass $\%$ or 15 at $\%$ than the solid solubility 0.1 mass $\%$ of nitrogen atoms in the equilibrium state. This high nitrogen solute concentration results in selective hardening of unmasked surface up to $1400 \mathrm{Hv}$. In the third, the sand-blasting with use of the optimum media works well to selectively remove the mold materials just below the printed masks. The original micro-dot pattern is accurately cut into the molds as a concave micro-dimple with right regular alignment. Since the nitrogen solute distributes only below the unmasked mold surface, the mold materials just below the masked region are selectively removed to make sharp-edged geometry as a micro-dimple. Finally, the injection molding is employed to demonstrate how accurately these micro-dimple textures are duplicated onto the PC cover case for cellular phone as a micro-disc pattern.

The authors would like to express their gratitude to Ms. B.M. Bouzan, Ms. F.P. Gusmo and Ms. L.U. Lobo da Silva (Brazil Federal Universities) during their stay at SIT, and Mr. T. Katoh (SIT) to their help in experiments, respectively. This study is financially supported in part by MITI with the contract of \#44001.

\section{References}

[1] I. Etsion, Tribology Letters, 17, 733-737 (2004)

[2] T. Aizawa, S. Satoh, T. Yamaguchi, Proc. 9th ICOMM, 15, 1-9 (2014)

[3] B. Denkena, J. Koehler, J. Laestner, Proc. 7th ICOMM, 2012; 85-89 (2012)

[4] Y. Jiang, W.S. Zhao, X.M. Kang, L. Gu, Proc. 6th ICOMM, 2011; 207-211 (2011)

[5] D. Santojoyo, T. Aizawa, S. Muraishi, H. Morita, Proc. 9th ICOMM, 90, 1-8 (2014)

[6] T. Aizawa, T. Yamaguchi, Proc. IWMF2014, 2a, 31-37 (2014)

[7] T. Katoh, T. Aizawa, T. Yamaguchi, Manufacturing Review, 2(2), 1-7 (2015)

[8] T. Aizawa, T. Yamaguchi, Proc. 10th 4M/ICOMM (2015) (in press)

[9] T. Aizawa, H. Suga, T. Katoh, L.U.L-Silva, T. Yamaguchi, Res. Rep. SIT. 52-1 (2015) (to be published)

[10] T. Aizawa, T. Yamaguchi, ICMCTF-2015, Abstract (2015) (in press) 